\title{
Clinical correlates of health-related quality of life among opioid-dependent patients
}

\author{
Kevin C. Heslin · Judith A. Stein · Keith G. Heinzerling • \\ Deyu Pan · Christine Magladry $\cdot$ Ron D. Hays
}

Accepted: 18 January 2011/Published online: 17 February 2011

(C) The Author(s) 2011. This article is published with open access at Springerlink.com

\begin{abstract}
Purpose Previous work suggests that opioid users have lower health-related quality of life (HRQOL) than patients with more prevalent chronic illnesses such as hypertension or diabetes. Although comparisons with population norms are informative, studies of the correlates of HRQOL for opioid users are needed to plan clinical services.

Methods We tested a conceptual model of the pathways between physiologic factors and symptoms in relation to HRQOL among 344 opioid users in a clinical trial. Physical and mental HRQOL were measured by the Short-Form (SF)-36; withdrawal signs, symptoms, and functioning were also measured with validated instruments. Using structural equation modeling, we tested hypotheses that medical history directly predicts withdrawal signs and symptoms, and that medical history, withdrawal signs and symptoms, and functioning predict the physical and mental HRQOL latent variables of the SF-36.

Results Most hypothesized relationships were significant, and model fit was good. The model explained $36 \%$ of the variance in mental HRQOL and $34 \%$ of the variance in physical HRQOL.

Conclusions The conceptual framework appears valid for explaining variation in the physical and mental HRQOL of opioid users undergoing medically managed withdrawal. Analysis of longitudinal data would help to evaluate more rigorously the adequacy of the model for explaining HRQOL in opioid withdrawal.
\end{abstract}

K. C. Heslin $(\bowtie)$ J. A. Stein · K. G. Heinzerling · D. Pan ·

C. Magladry $\cdot$ R. D. Hays

Los Angeles, CA, USA

e-mail: kevin.heslin@va.gov
Keywords Opiate dependence - Quality of Life · Models, Structural · Trials, Randomized clinical

\section{Introduction}

Opioid dependence is a chronic disorder with multi-faceted negative medical, psychological, and social consequences [1]. Outcomes of treatment for opioid dependence include reductions or cessation in opioid use, typically assessed via self-reported drug use and laboratory tests for opioid metabolites in urine. But increasingly there have been calls for the development of additional outcome measures to capture treatment-related changes in the medical, psychological, and social domains impacted by addiction [2]. Patient reports of their functioning and well-being-i.e., their health-related quality of life (HRQOL) - are used increasingly as outcome measures in the evaluation of care for chronic disorders [3], and measures of HRQOL are strong predictors of mortality, disease complications, and resource use [4]. As a result, measures of HRQOL may provide an important tool for assessing outcomes of treatment for opioid dependence.

The Short-Form 36 (SF-36) is the most widely administered HRQOL instrument in health outcomes research [5]. The SF-36 measures aspects of health relevant for adults in general, allowing for comparisons between patients with a wide range of health problems [6-12]. In a number of studies, opioid users in treatment and community settings have had consistently lower average SF-36 scores, compared with both the general population and the patients with serious chronic illnesses such as diabetes and hypertension [13-21, 23, 24]. For example, an Australian study of symptom-triggered taper methods for benzodiazepine withdrawal found that patients scored substantially 
lower on all eight SF-36 scales than did the general population [21]. Compared with U.S. data on patients with serious medical conditions, opioid users in a Canadian methadone program had lower SF-36 scores on social functioning, emotional well-being, energy/vitality, and role limitations due to emotional health problems [22].

Comparisons of HRQOL between opioid-dependent patients and the general population are informative; however, studies of the clinical determinants of HRQOL specifically among these patients are also needed for planning and evaluating detoxification and other treatment programs. A study in North Carolina examined determinants of HRQOL in a convenience sample of active injection drug users, $82 \%$ of whom reported recent heroin use. Results from eight multiple regression models-one for each of the eight SF-36 scales-identified negative associations of HRQOL with self-reports of harmful drinking, heroin use, and methamphetamine use [23].

In this study, we test a conceptual framework of the direct and mediated pathways between HRQOL, addiction severity, withdrawal symptoms, and physiologic factors among patients in treatment for opioid dependence. The evaluation of such a framework is important for identifying relationships between clinical variables and HRQOL that can be used to guide treatment and optimize outcomes for this patient population. We hypothesized that worse withdrawal symptoms and addiction severity within our patient population would predict the reports of poorer physical and mental HRQOL (Fig. 1).

\section{Methods}

\section{Participants}

We analyzed baseline data on 344 subjects in a multicenter trial of buprenophrine conducted in the National Institute on Drug Abuse Clinical Trials Network (CTN). Recruitment was conducted from January 2001 to February 2002. Eligible individuals were at least 18 years old, met diagnostic criteria for opioid dependence, and needed medical management for withdrawal. Exclusion criteria included serious medical or psychiatric conditions that would make participation hazardous, as well as pregnancy, current lactation, and plans to become pregnant. A total of 113 inpatients and 231 outpatients participated at any of 12 community-based treatment programs in the CTN. Further details on study design and implementation are available elsewhere [24]. Our secondary analyses of these public use data were exempted from review by the Institutional Review Board at the Charles Drew University of Medicine and Science.

\section{Conceptual framework}

The Wilson and Cleary model positions overall quality of life at the endpoint of a multi-level continuum, with a domain of physiologic variables as the starting point [25]. The physiologic domain includes the type of clinical information gathered in laboratory tests, physical examinations, and medical histories. Physiologic factors are hypothesized to influence a domain of signs and symptoms, which refer, respectively, to clinicians' observations and patients' perceptions of abnormal physical, emotional, and cognitive states. Signs and symptoms directly predict functioning, which in turn predicts general health perceptions and finally overall quality of life. In this analysis, we modified the Wilson-Cleary model so that general health perceptions were represented by physical and mental health. Although the main causal relationships in this framework are hypothesized to exist between adjacent domains (e.g., physiologic factors and symptoms), it is also possible for domains to exert direct effects on nonadjacent domains (e.g., physiologic factors and functioning). Because we did not include a measure of overall quality of life in this study, we were unable to examine the end of the continuum in the analysis.

\section{Measures}

Five of the constructs in our modification of the WilsonCleary model are represented as latent variables: physical
Fig. 1 Conceptual framework (schematic) linking clinical variables and health-related quality of life. HRQOL, healthrelated quality of life. Reference: Wilson and Cleary [25]

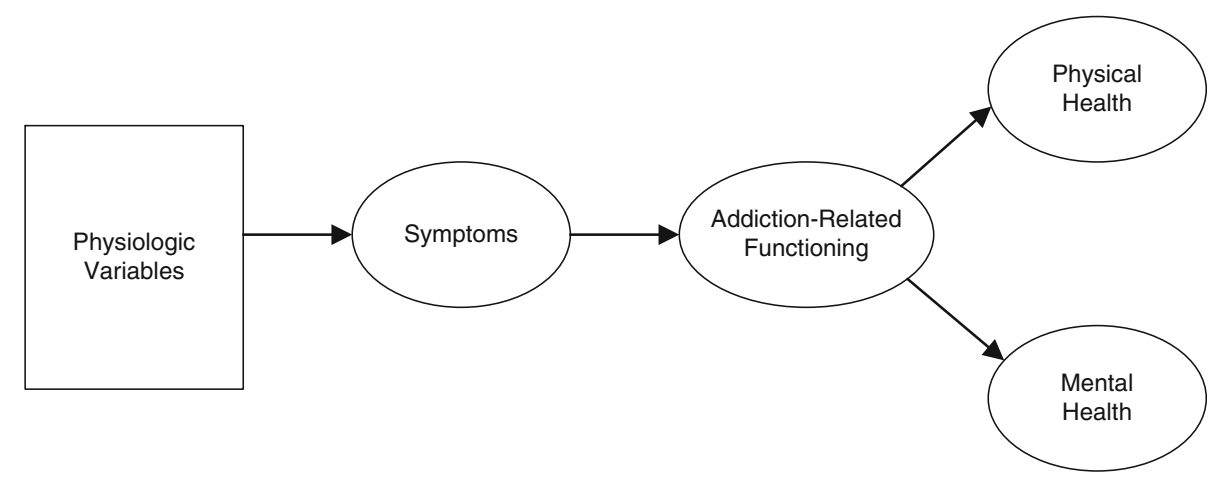


health, mental health, opioid withdrawal signs (physician observed), opioid withdrawal symptoms (patient reported), and addiction severity. A latent variable is an underlying unobserved construct that is indicated by two or more measured variables [26]. The latent variables in this analysis were created from multi-item scales described below.

\section{Self-reported physical and mental health}

The physical and mental health latent variables were defined by the eight scales of the SF-36 V.1. Four scales are associated primarily with physical health, and four scales are associated primarily with mental health. Previous research indicates that the SF-36 indicators of physical and mental health are positively correlated [27]. We calculated the eight SF-36 scale scores for (1) physical health: physical functioning (ten items); role limitations due to physical health problems (four items); bodily pain (two items); and general health perceptions (five items); and (2) mental health: social functioning (two items); emotional wellbeing (five items); role limitations due to emotional problems (three items); and energy/fatigue (four items). Twenty items use a "past 4 weeks" recall period, and 15 items do not have a recall period; the "health transition" item is not used in scoring any of the eight scales. Each of the eight scale scores were scored with a T-score metric using 1998 U.S. general population means and standard deviations (mean $=50, \mathrm{SD}=10$ ) [28]. The scale scores were then allowed to load on two factors representing physical and mental health.

\section{Adjective rating scale of withdrawal (ARSW)}

Baseline opioid withdrawal symptoms were assessed using the ARSW [29]. Patients rated themselves on response scales ranging from 0 ("none") to 9 ("severe") for the following 16 signs and symptoms of withdrawal: muscle cramps, depressed or sad, painful joints, excessive yawning, hot or cold flashes, trouble getting to sleep, nausea, irritability, runny nose, poor appetite, weak knees, excessive sneezing, tense and jittery, watery eyes, abdominal cramps, and fitful sleep. With such a broad range of scores, we assumed an underlying continuous distribution of this variable. Because of the high coefficient alpha among these indicators (.96), and to avoid too many indicators for the size of the patient sample, the 16 items were randomly combined into four "parcels" [30]. For this variable, a "parcel" refers to an average of the responses of four randomly selected ARSW items; thus, we included four parcels, each containing four of the 16 ARSW items, in the model. Parceling is acceptable in structural modeling when alpha coefficients are high [31] and when sets of items are sufficiently unidimensional so that important features of individual items are not discarded when they are combined [32]. In this sample, coefficient alpha was exceptionally high (.96). Parceling typically results in better model fit than do models using individual items with their attendant measurement error. Models were also tested without parceling and were highly similar to those reported in this article. However, due to the sample size, they are not presented because they had too many parameters to be estimated reliably.

\section{Clinical opiate withdrawal scale (COWS)}

Baseline opioid withdrawal signs were assessed using the COWS. The COWS is an 11-item instrument designed to provide a description of the following signs and symptoms of withdrawal that can be directly observed by a physician interviewer: increased resting pulse rate, gastrointestinal upset, sweating, tremor (observation of outstretched hands), restlessness, yawning, pupil size (extent of dilation), anxiety or irritability, arthralgias, piloerection of skin, and runny nose or tearing (not accounted for by cold symptoms or allergies). Responses to the 11 items are summed to construct a COWS score that can range from 0 to 48 , with higher scores indicating greater withdrawal severity [33]. Due to considerations cited above, the 11 items were randomly combined into four parcels (coefficient alpha $=.77$ ). Three of the parcels consisted of three items, and one parcel consisted of two items.

\section{Addiction severity index (ASI) lite psychiatric and family/social scales}

The ASI Lite was developed to assess patient functioning in seven problem areas commonly affected by substance use disorders. To represent the functioning domain of the Wilson-Cleary framework for this patient population, we used the Psychiatric and Family/Social scales of the instrument. Composite scores for problems in each of these two domains in the previous 30 days were calculated using procedures described by McGahan and colleagues [34]. The Psychiatric composite score includes 11 items assessing aspects of current psychiatric status and functioning such as hallucinations; "serious anxiety or tension;" and cognitive dysfunction ("trouble understanding, concentrating, or remembering"). The Family/Social composite score is primarily a measure of psycho-social functioning. It includes five items assessing the presence of conflict with family, friends, co-workers, neighbors, and others; the respondent's satisfaction with his or her current marital/relationship status, and the degree to which the respondent was "troubled or bothered" by family problems. 


\section{Physiologic measures}

Opioid-dependent patients are likely to differ in their underlying risks for severe withdrawal signs and symptoms, resulting from factors such as the presence of coexisting conditions and medical history. For this reason, we included data from the CTN baseline medical history and physical examination; the findings of which were recorded on standardized reporting forms by the clinician conducting the histories and examinations. We created a count variable by summing the total number of health conditions that clinicians elicited from patients during medical history-taking, from a list of 17 conditions affecting organs or organ systems such as the eyes, ears, nose and throat; the cardiovascular system; the integumentary system; and the liver. We created another count variable representing the total number of abnormal findings from a physical exam, from a list of 17 organ systems and body parts such as the abdomen, lymph nodes, lungs, pelvis, and genitalia. Because a recent study showed an association between HRQOL and body mass index (BMI) [35], we also included patient $\mathrm{BMI}$ in the physiologic domain of the model.

\section{Hypotheses}

We hypothesized that BMI, abnormal physical exam findings, and medical history would predict variation in withdrawal signs and symptoms - which, in turn, were hypothesized to predict functioning as measured by the ASI Psychiatric and Family/Social scales. Significant correlations were hypothesized between physical exam findings and medical history. Finally, we hypothesized that the ARSW, COWS, ASI scales, and medical history would predict self-reported physical and mental health.

\section{Statistical analysis}

We used the EQS 6 structural equation modeling (SEM) program [36], which compares a proposed hypothetical model with a set of actual data. The closeness of the variance-covariance matrix implied by the hypothetical model to the empirical data was evaluated using the maximum likelihood chi-squared statistic (ML $\chi^{2}$ ) and two goodnessof-fit indices: The comparative fit index (CFI) and the Root Mean Square Error of Approximation (RMSEA). Comparative fit index values of .95 or greater are desirable and indicate good fit. The RMSEA is a measure of lack of fit per degrees of freedom, controlling for sample size. Values for the RMSEA less than .06 indicate a close-fitting model [37]. Robust statistics (Robust CFI and Satorra-Bentler $\chi^{2}$ ) correcting for multivariate non-normality were also examined, because the multivariate kurtosis estimate was high (Mardia's normalized estimate $=12.96$ ). We also report the Yuan-Bentler residual-based chi-square statistic, which performs well in small samples when robust statistics are more appropriate [38, 39].

Due to the continuous and broad variations in most of the data, we assumed a continuous underlying distribution in our choice of statistical methods. Nonsignificant paths and covariances were incrementally dropped until only significant paths and covariances remained. To improve model fit, we examined the results of the Lagrange Multiplier (LM) Test, which suggests additional significant parameters to be added to the model [40]. Indirect effects were also assessed [41].

\section{Results}

Table 1 provides sociodemographic information for the study sample. Table 2 reports summary statistics for all variables included in the model and factor loadings of the indicators for the latent variables in the confirmatory factor analysis (CFA). Table 3 reports correlations among the variables in the CFA before the hypothesized directional path model was tested. The CFA tested the viability of the proposed factor structure of the latent variables and provided correlations among components of the model. As expected, the physiologic and symptom variables generally had stronger associations with the physical health score than with the mental health score (Table 3).

The final predictive structural equation model is presented in Fig. 2. Measured variables are depicted in rectangles and the latent variables are in circles. Most hypothesized relationships were significant. One reasonable supplementary relationship suggested by the LM Test was added to improve model fit, i.e., a correlated residual between emotional wellbeing and energy/fatigue $(r=.43)$. Model fit was good: ML $\chi^{2}=379.37,176 d f$; CFI $=.95$, RMSEA $=.06$; SatorraBentler Robust $\chi^{2}=356.19,176 d f ; \quad$ RCFI $=.95$, RMSEA $=.056$; Yuan-Bentler $\chi^{2}=217.24$. This model

Table 1 Sociodemographic characteristics of opioid-dependent patients $(n=344)$

\begin{tabular}{lll}
\hline & $\%(n)$ & Mean (SD) \\
\hline Race & & \\
African-American/Black & $30.9(106)$ & - \\
Hispanic/Latino & $18.7(64)-$ \\
White & $45.2(155)-$ \\
Other & $5.3(18)-$ & - \\
Female gender & $32.5(111)$ & - \\
Age & - & $37.9(10.2)$ \\
Years of formal education & - & $12.5(1.9)$ \\
Income (\$), all sources, past 3 months & - & $1732.9(1938.3)$ \\
\hline
\end{tabular}


Table 2 Descriptive statistics, internal consistency estimates, and factor loadings for health-related characteristics of opioid-dependent patients $(n=344)$

Mean (SD) Alpha $\quad$ Loading

Health-related quality of life

Physical Health (SF-36 T scores)

Physical functioning

$\begin{array}{lll}50.3(9.0) & 0.91 & 0.73 \\ 44.7(9.7) & 0.75 & 0.71 \\ 47.1(12.0) & 0.86 & 0.82 \\ 44.6(12.3) & 0.90 & 0.73 \\ & & \\ 38.4(12.1) & 0.81 & 0.80 \\ 38.7(12.2) & 0.76 & 0.88 \\ 44.1(10.5) & 0.81 & 0.72 \\ 39.5(14.2) & 0.89 & 0.74\end{array}$

General health perceptions

Bodily pain

Role limitations due to physical health problems

Mental Health (SF-36 T scores)

Emotional well-being

Social functioning

Energy/fatigue

Role limitations due to emotional problems

$39.5(14.2)$

Withdrawal signs and symptoms

$$
\begin{aligned}
& \text { ARSW "Parcel 1" } \\
& \text { ARSW "Parcel 2"a } \\
& \text { ARSW "Parcel 3" } \\
& \text { ARSW "Parcel 4" } \\
& \text { COWS "Parcel 1" } \\
& \text { COWS "Parcel 2" } \\
& \text { COWS "Parcel 3"b } \\
& \text { COWS "Parcel 4" }
\end{aligned}
$$

$0.56(0.54)$

$0.14(0.18)$

$0.18(0.19)$

$25.2(4.8)$

Count of abnormal findings on physical exam

Count of positive findings from medical history

$\begin{array}{ll}- & .92 \\ - & .90 \\ - & .93 \\ - & .95 \\ - & .61 \\ - & .76 \\ - & .60 \\ - & .80 \\ & \\ .63 & .55 \\ .80 & .81\end{array}$

Physiologic factors
0.89

0.74

0.73

0.71

.73

.80

88

74

92

90

93

60

80

55

\begin{tabular}{|c|c|c|c|c|c|c|c|c|c|}
\hline & & 1. & 2. & 3. & 4. & 5. & 6. & 7. & 8. \\
\hline 1. & BMI & - & & & & & & & \\
\hline 2. & Exam findings & .09 & - & & & & & & \\
\hline 3. & Medical history & .01 & $.31 * * *$ & - & & & & & \\
\hline 4. & Withdrawal Signs & -.07 & .02 & $.22 * * *$ & - & & & & \\
\hline 5. & Withdrawal Symptoms & $-.16^{* *}$ & -.03 & $.28 * * *$ & $.56 * * *$ & - & & & \\
\hline 6. & ASI Psych-Family & .04 & $.14^{*}$ & $.19 * *$ & $.15^{*}$ & $.23 * * *$ & - & & \\
\hline 7. & Physical HRQOL & .00 & $-.19 * * *$ & $-.35 * * *$ & $-.28 * * *$ & $-.49 * * *$ & $-.32 * * *$ & - & \\
\hline 8. & Mental HRQOL & .04 & $-.14 *$ & $-.26 * * *$ & $-.19 * *$ & $-.31 * * *$ & $-.55 * * *$ & $.84 * * *$ & - \\
\hline
\end{tabular}

81

$\begin{array}{ll}- & - \\ - & - \\ - & -\end{array}$

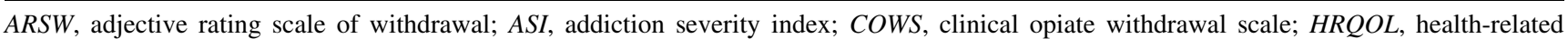
quality of life; $S F-36$, short-form 36

a The 16 items of the ARSW were randomly combined into "parcels" to obtain mean indicators

b The 11 items of the COWS were randomly combined into "parcels" to obtain mean indicators

Table 3 Correlations among variables in the confirmatory factor analysis (CFA)

$* p \leq .05$, ** $p \leq .01, * * * p \leq .001$. Withdrawal signs assessed with COWS, Clinical Opiate Withdrawal Scale. Withdrawal symptoms assessed with ARSW, Adjective Rating Scale of Withdrawal. ASI Psych-Family, Addiction severity index psychiatric and family functioning scales; $B M I$, body mass index; $H R Q O L$, health-related quality of life 


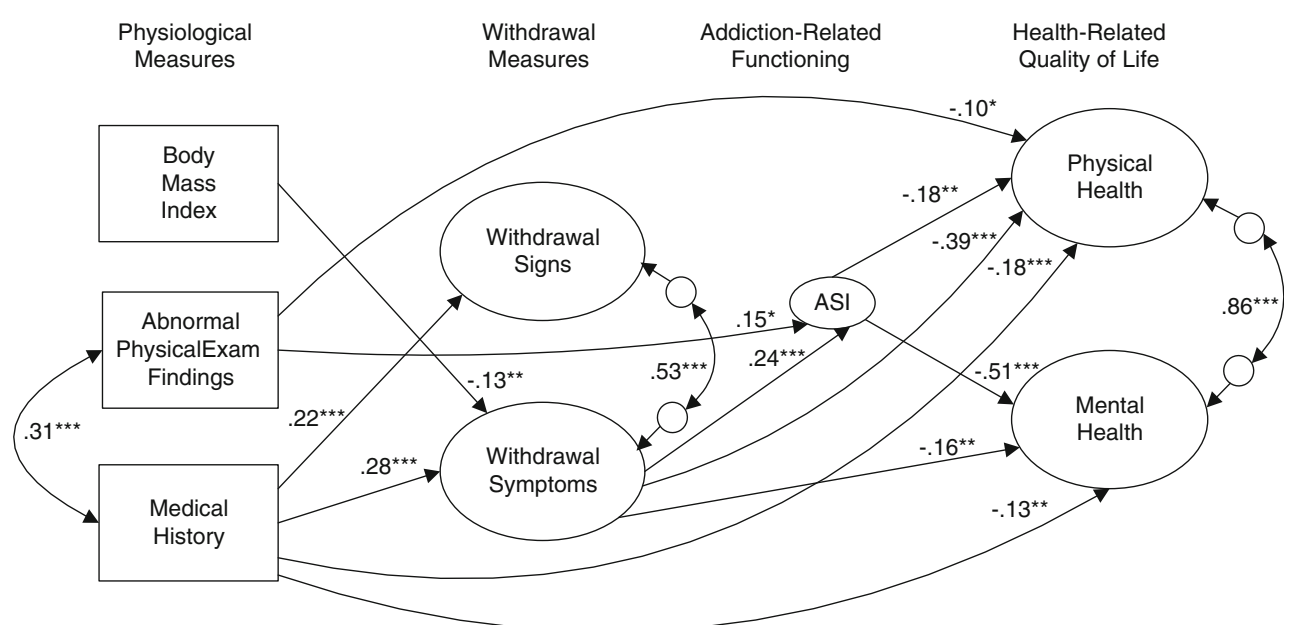

Fig. 2 Structural equation model depicting significant regression paths $(n=344)$. Ovals represent latent variables; rectangles represent single-item indicators; small circles represent correlated error terms. Single-headed arrows represent regression coefficients; double-headed arrows represent correlations between predictive background variables and correlations between residuals of dependent

explains $36 \%$ of the variance in mental health and $34 \%$ of the variance in physical health.

The structural equation model identified a number of indirect and direct effects among variables representing the domains of the conceptual model, going from the physiologic level to physical and mental HRQOL (i.e., from left to right in Fig. 2). Among the direct effects, a greater BMI predicted fewer withdrawal symptoms. A greater number of health conditions taken from medical histories predicted more withdrawal signs and symptoms, as well as lower physical health and mental health. A greater number of abnormal findings from physical exams predicted lower physical health and more impaired functioning on the ASI scales. Withdrawal signs were strongly associated with withdrawal symptoms ( $r=.56$ in the CFA) but did not independently predict any of the variables further along the continuum of the conceptual framework; further, its influence was attenuated by its association with withdrawal symptoms (which did strongly predict a higher ASI, and lower QOL measures). Finally, the psychiatric and family/ social functioning scales of the ASI latent variable predicted lower physical and mental health.

Significant indirect effects influencing variables through their effects on the intermediate predictors include a significant negative indirect effect of BMI on ASI $(p<.05)$ and a positive effect of a poor medical history on ASI $(p<.01)$. These effects were mediated through withdrawal symptoms-which, in turn, predicted ASI. Indirect effects also had an impact on the physical and mental health latent variables. Physical health was indirectly impacted by BMI (positive, $p<.01$ ), and negatively by a poor medical history $(p<.001)$ through their influence on withdrawal variables. Withdrawal signs were based on physician observation (with the Clinical Opiate Withdrawal Scale). Withdrawal symptoms were based on patient report (with the Adjective Rating Scale of Withdrawal). ASI, addiction severity index (Psychiatric and Family/ Social scales). Regression coefficients are standardized $(* p \leq .05$, $* * p \leq .01, * * * p \leq .001)$

symptoms. Withdrawal symptoms $(p<.05)$ also had a significant indirect effect mediated through ASI. Mental health was impacted indirectly by BMI (positively, $p<.01$ ) and poor medical history (negatively, $p<.001$ ) through withdrawal symptoms. Abnormal physical exam findings $(p<.05)$ and withdrawal symptoms $(p<.001)$ negatively impacted mental health through their influence on ASI. These influences can be traced on Fig. 2.

\section{Discussion}

A number of studies have used the Wilson-Cleary framework to analyze data on patients with conditions as diverse as xerostomia [6], HIV disease [7, 8], heart disease [9, 10], and Hodgkin's lymphoma [11]. To our knowledge, no previous study has used this conceptual framework to analyze HRQOL among patients with a primary diagnosis of opioid dependence or any other substance use disorder. Results from the current study suggest that the modification of the Wilson-Cleary framework evaluated here accounts for meaningful variation in physical and mental health in this patient population. Because the baseline CTN data are cross-sectional, the conceptual framework is not proven by this analysis; however, the hypothesized relationships among variables that we specified are consistent with the data.

Previous analyses of HRQOL among opioid users have not been based on an established conceptual framework of health and a parsimonious theory-based statistical model such as the one used in the current study. A study of a predominantly heroin-using sample reported results from 
eight multiple regression models of the SF-36 scales, which raises potential problems with multiple statistical testing [23]. The physical and mental latent variables have a number of analytic advantages over a focus on the eight scales, including generally smaller confidence intervals and a reduction in the number of statistical comparisons [42].

Greater self-reported withdrawal symptoms predicted lower HRQOL, directly and via greater addiction severity. Greater opioid withdrawal symptoms likely reflect heavier opioid use and higher opioid dependence, which may explain the negative association with HRQOL. In addition, self-reported withdrawal symptoms were related to HRQOL, while physician-observed withdrawal signs (COWS) were not. Since HRQOL taps patients' subjective well-being, it may be that patients' perceptions of withdrawal severity are more important for HRQOL than objective assessments of withdrawal via clinicians. The patient-reported measures may have drawn on both the sensory aspect of symptoms and the respondent's emotional responses to them, whereas clinician ratings are limited to a relatively narrow set of observable patient behaviors. The decreased physical and mental health associated with withdrawal symptoms underscore the importance of treating these symptoms during acute withdrawal. Approved pharmacologic treatments for opioid dependence such as buprenorphine and methadone are effective in reducing illicit opioid use and in relieving opioid withdrawal symptoms [43, 44] and may improve HRQOL via reductions in opioid withdrawal symptoms.

An advisory from the Substance Abuse and Mental Health Services Administration notes that patients often need monitoring and medical management for symptoms of "protracted withdrawal," which refers to the persistence of substance-specific withdrawal symptoms for weeks, months, or even years after the generally expected timeframe for acute withdrawal has ended [45]. Protracted symptoms such as fatigue, dysphoria, and unexplained physical complaints would likely contribute to poorer physical and mental HRQOL, and may eventually lead patients to seek relief by returning to substance use. By adding HRQOL measures to inform ongoing treatment decisions, clinicians may become more effective at identifying therapies that improve areas of health that matter greatly to patients, particularly to those experiencing protracted withdrawal symptoms. Randomized studies in general medicine and oncology suggest that the integration of HRQOL measures into routine practice leads to improvements in processes of care (for example, physician-patient communication) [46]; however, to our knowledge, addiction medicine researchers have not studied the feasibility and potential benefits of using HRQOL measures in regular clinic practice. This is a potential area for future work.
Several limitations of the study should be discussed. The sample was enrolled in a multi-center study conducted in the National Institute on Drug Abuse CTN and included both ambulatory care patients and hospitalized patients; a study focused solely on patients seen in one of these settings may have generated different results. The generalizability of clinical trial findings to opioid users in the community may be limited; however, the physiologic signs and symptoms of opioid withdrawal probably do not differ greatly across these populations. Twenty items of the SF-36 use a "past 4 weeks" recall period, whereas the ARSW items use a 24-h recall period-which makes using the ARSW as a predictor of the PCS and MCS problematic. However, because one would expect a more "acute" version of the SF-36 to be more sensitive to variation in acute symptoms [47], any bias resulting from differences in recall periods between the ARSW and SF-36 would likely be toward the null.

Our sample size of 344 was small for the application of structural equation modeling, which is why we used the eight SF-36 V.1 scales instead of the 35 separate items. In a previous work, the SF-36 energy/fatigue scale has been allowed to load on both the physical and the mental health factors [28]; we specified that this scale load only on the mental health factor. We also used item parcels for the ARSW and COWS because of the sample size. We ran an alternative model without parceling the items for these scales; however, with 344 subjects and over 100 free parameters, the results were unreliable; however, for the alternative model, the ML $\chi^{2}$ statistic was 1135.34 on 665 degrees of freedom, RCFI was .934, and RMSEA was .05. Although parceling may be justifiable if the parceled items represent a single construct, the technique obscures any unique information attributable to individual items. If items represent different facets of the same construct, then improvements in model fit due to parceling would be misleading [48]. The statistical procedures used presumed the indicators (items or parcels) to have a continuous underlying distribution. We used robust estimators (Robust CFI and Satorra-Bentler $\chi^{2}$ ) to allow for distributional asymmetry in the data; however, these estimators assume that indicators are measured on an interval scale, and it is debatable whether ordinal items should be treated as interval data. More robust estimators could have been applied if a larger sample had been available. Results must also be considered in light of the limitations of fitting a model of a temporal process to cross-sectional data. Analysis of longitudinal data with cross-lagged panel designs or latent curve models would help to evaluate more rigorously the effects of symptoms and other antecedent variables on HRQOL.

The results from this analysis suggest that physiologic factors have demonstrable effects on withdrawal symptoms 
and physical and mental HRQOL among opioid users. Current approaches to substance abuse and other psychiatric disorders depend greatly on the patient's reported experience of illness. The practice of "measurement-based care" refers to the systematic measurement of symptoms and side effects at each treatment visit and the use of guidelines to modify therapy based on patient-reported measures [49]. Within opioid dependence treatment specifically, amelioration of opioid withdrawal symptoms using available pharmacotherapies such as buprenorphine or methadone may improve HRQOL in addition to reducing illicit opioid use. One benefit of using HRQOL instruments in measurement-based care is that they are outcomes that are important to patients [50]. Future work should assess whether opioid dependence treatments that are informed by the effects of treatment on HRQOL lead to better outcomes, including increases in patients' motivation to engage in longer-term rehabilitation efforts.

Acknowledgments This work was supported by the National Institute on Drug Abuse (1R03-DA018762-01A2 and 1P01-DA01070-37). Kevin Heslin was supported in part by the VHA Emergency Management Evaluation Center. Keith Heinzerling was supported by NIDA grant 1 K23 DA 023558. Ron Hays was supported in part by UCLA/DREW Project EXPORT (NCMHD, 2P20MD000182) and the UCLA Resource Center for Minority Aging Research/Center for Health Improvement in Minority Elderly (NIA, P30AG021684).

Open Access This article is distributed under the terms of the Creative Commons Attribution Noncommercial License which permits any noncommercial use, distribution, and reproduction in any medium, provided the original author(s) and source are credited.

\section{References}

1. Strain, E. C., \& Stitzer, M. L. (2006). The treatment of opioid dependence (1st ed.). Baltimore: Johns Hopkins University Press.

2. Miller, P. G., \& Miller, W. R. (2009). What should we be aiming for in the treatment of addiction? Addiction, 104(5), 685-686.

3. Stewart, A. L., Sherbourne, C. D., Hays, R. D., Wells, K. B., Nelson, E. C., Kamberg, C. J., et al. (1992). Summary and discussion of MOS measures. In A. L. Stewart \& J. E. Ware (Eds.), Measuring functioning and well-being: The medical outcomes study approach (pp. 345-371). Durham, NC: Duke University Press.

4. Bjorner, J., Fayers, P., \& Idler, E. (2004). Self-rated health. In P. Fayers \& R. D. Hays (Eds.), Assessing quality of life in clinical trials (pp. 309-324). New York: Oxford University Press.

5. McDowell, I. (2006). Measuring health: A guide to rating scales and questionnaires. New York: Oxford University Press.

6. Baker, S. R., Pankhurst, C. L., \& Robinson, P. G. (2007). Testing relationships between clinical and non-clinical variables in xerostomia: A structural equation model of oral health-related quality of life. Quality of Life Research, 16, 297-308.

7. Sousa, K. H., \& Kwok, O. M. (2006). Putting Wilson and Cleary to the test: Analysis of a HRQOL conceptual model using structural equation modeling. Quality of Life Research, 15, 725-737.
8. Vidrine, D. J., Amick, B. C., Gritz, E. R., \& Arduino, R. C. (2005). Assessing a conceptual framework of health-related quality of life in a HIV/AIDS population. Quality of Life Research, 14, 923-933.

9. Janz, N. K., Janevic, M. R., Didge, J. A., et al. (2001). Factors influencing quality of life in older women with heart disease. Medical Care, 39, 588-598.

10. Heo, S., Moser, D. K., Riegel, B., Hall, L. A., \& Christman, N. (2005). Testing a published model of health-related quality of life in heart failure. Cardiac Failure, 11, 372-379.

11. Wettergren, L., Björkholm, M., Axdorph, U., \& Langius-Eklöf, A. (2004). Determinants of health-related quality of life in longterm survivors of Hodgkin's lymphoma. Quality of Life Research, 13, 1369-1379.

12. Baker, S. R., Pankhurst, C. L., \& Robinson, P. G. (2007). Testing relationships between clinical and non-clinical variables in xerostomia: A structural equation model of oral health-related quality of life. Quality of Life Research, 16, 297-308.

13. Ryan, C. F., \& White, J. M. (1996). Health status at entry to methadone maintenance treatment using the SF-36 health survey questionnaire. Addiction, 91, 39-45.

14. Neale, J. (2004). Measuring the health of Scottish drug users. Health and Social Care in the Community, 12, 202-211.

15. Perneger, T. V., Giner, F., del Rio, M., \& Mino, A. (1998). Randomised trial of heroin maintenance programme for addicts who fail in conventional drug treatments. $B M J, 317,13-18$.

16. Richter, D., Eikelmann, B., \& Berger, K. (2004). Use of the SF36 in the evaluation of a drug detoxification program. Quality of Life Research, 13, 907-914.

17. Freeman, K. (2003). Health and well-being outcomes for drugdependent offenders on the NSW Drug Court programme. Drug and Alcohol Review, 22, 409-416.

18. Deering, D. E., Frampton, C. M. A., Horn, J., Sellman, J. D., Adamson, S. J., \& Potiki, T. L. (2004). Health status of clients receiving methadone maintenance treatment using the SF-36 health survey instrument. Drug and Alcohol Review, 23, 273-280.

19. Dalgard, O., Egeland, A., Skaug, K., Vilimas, K., \& Steen, T. (2004). Health-related quality of life in active injecting drug users with and without chronic hepatitis $\mathrm{C}$ virus infection. Hepatology, $39,74-80$.

20. Kertesz, S. G., Larson, M. J., Horton, N. J., Winter, M., Saitz, R., \& Samet, J. H. (2005). Homeless chronicity and health-related quality of life trajectories among adults with addictions. Medical Care, 43, 574-585.

21. McGregor, C., Machin, A., \& White, J. M. (2003). In-patient benzodiazepine withdrawal: Comparison of fixed and symptomtriggered taper methods. Drug and Alcohol Review, 22, 175-180.

22. Millson, P. E., Challacombe, L., Villeneuve, P. J., Fischer, B., Strike, C. J., Myers, T., et al. (2004). Self-perceived health among Canadian opiate users: A comparison to the general population and to other chronic disease populations. Canadian Journal of Public Health, 95, 99-103.

23. Costenbader, E. C., Zule, W. A., \& Coomes, C. M. (2007). The impact of illicit drug use and harmful drinking on quality of life among injection drug users at high risk for hepatitis $\mathrm{C}$ infection. Drug and Alcohol Dependence, 89, 251-258.

24. Ling, W., Amass, L., Shoptaw, S., et al. (2005). A multi-center randomized trial of buprenorphine-naloxone versus clonidine for opioid detoxication: Findings from the National Institute on Drug Abuse Clinical Trials Network. Addiction, 100, 1090-1100.

25. Wilson, I. B., \& Cleary, P. D. (1995). Linking clinical variables with health-related quality of life. A conceptual model of patient outcomes. JAMA, 273, 59-65.

26. Bentler, P. M., \& Stein, J. A. (1992). Structural equation models in medical research. Statistical Methods in Medical Research, 1, 159-181. 
27. Ware, J. E., Kosinski, M., \& Dewey, J. E. (2000). How to score version two of the SF-36 health survey (Vol. 1). Lincoln, RI: QualityMetric, Incorporated.

28. Hays, R. D., Marshall, G. N., Wang, E. Y. I., \& Sherbourne, C. D. (1994). Four-year cross-lagged associations between physical and mental health in the Medical Outcomes Study. Journal of Consulting and Clinical Psychology, 62, 441-449.

29. Amass, L., Kamien, J. B., \& Mikulich, L. K. (2000). Efficacy of daily and alternate-day dosing regimens with the combination buprenorphine-naloxone tablet. Drug and Alcohol Dependence, 58, 143-152.

30. Little, T. D., Cunningham, W. A., Shahar, G., \& Widaman, K. F. (2002). To parcel or not to parcel: Exploring the question, weighing the merits. Structural Equation Modeling, 9, 151-173.

31. Yuan, K.-H., Bentler, P. M., \& Kano, Y. (1997). On averaging variables in a confirmatory factor analysis model. Behaviormetrika, 24, 71-83.

32. Bandalos, D. L., \& Finney, S. J. (2001). Item parceling issues in structural equation modeling. In G. A. Marcoulides (Ed.), New developments and techniques in structural equation modeling (pp. 269-296). Mahwah, NJ: Lawrence Erlbaum \& Associates.

33. Wesson, D. R., \& Ling, W. (2003). The clinical opiate withdrawal scale (COWS). Journal of Psychoactive Drugs, 35, 253-259.

34. McGahan, P. L., Griffith, J. A., Parente, R., \& McLellan, A. T. (1986). Addiction severity index composite scores manual. Philadelphia, PA: Treatment Research Institute.

35. Søltoft, F., Hammer, M., \& Kragh, N. (2009). The association of body mass index and health-related quality of life in the general population: Data from the 2003 Health Survey of England. Quality of Life Research, 18, 1293-1299.

36. Bentler, P. M. (2006). EQS 6 structural equations program manual. Encino, CA: Multivariate Software, Inc.

37. Hu, L., \& Bentler, P. M. (1999). Cutoff criteria for fit indexes in covariance structural analysis: Conventional criteria versus new alternatives. Structural Equation Modeling, 6, 1-55.

38. Yuan, K.-H., \& Bentler, P. M. (1998). Robust mean and covariance structure analysis. British Journal of Mathematical and Statistical Psychology, 51, 63-88.

39. Yuan, K.-H., \& Bentler, P. M. (1999). On asymptotic distributions of normal theory MLE in covariance structure analysis under some nonnormal distributions. Statistics \& Probability Letters, 42, 107-113.

40. Chou, C., \& Bentler, P. M. (1990). Model modification in covariance structure modeling: A comparison among likelihood ratio, Lagrange multiplier, and Wald tests. Multivariate Behavioral Research, 25, 115-136.

41. Sobel, M. E. (1987). Direct and indirect effects in linear structural equation models. Sociological Methods \& Research, 16, $155-176$.

42. Ware, J. E., Gandek, B., Kosinski, M., Aaronson, N. K., Apolone, G., Brazier, J., et al. (1998). The equivalence of SF-36 summary health scores estimated using standard and country-specific algorithms in 10 countries: Results from the IQOLA Project. Journal of Clinical Epidemiology, 51, 1167-1170.

43. Gowing, L., Ali, R., \& White, J. M. (2009). Buprenorphine for the management of opioid withdrawal. Cochrane Database Systematic Review; CD002025.

44. Amato, L., Davoli, M., Minozzi, S., Ali, R., \& Ferri, M. (2005). Methadone at tapered doses for the management of opioid withdrawal. Cochrane Database Systematic Review; CD003409.

45. Center for Substance Abuse Treatment. Protracted Withdrawal. Substance Abuse Treatment Advisory, Volume 9, Issue 1.

46. Velikova, G., \& Wright, P. (2005). Individual patient monitoring. In Fayer P, Hays R, (Eds.) Assessing quality of life in clinical trials. Oxford University Press.

47. Keller, S. D., Bayliss, M. S., Ware, J. E., Hsu, M. A., Damiano, A. M., \& Goss, T. F. (1997). Comparison of responses to SF-36 health survey questions with one-week and four-week recall periods. Health Services Research, 32, 367-384.

48. Bandalos, D. L. (2002). The effects of item parceling on goodness-of-fit and parameter estimate bias in structural equation modeling. Structural Equation Modeling, 9, 78-102.

49. Trivedi, M. H., Rush, A. J., Wisniewski, S. R., et al. (2006). Evaluation of outcomes with Citalopram for depression using measurement-based care in STAR*D: Implications for clinical practice. American Journal of Psychiatry, 163, 28-40.

50. Reiser, S. J. (1993). The era of the patient: Using the experience of illness in shaping the missions of health care. JAMA, 269, 1012-1018. 